\title{
CONSIDERAÇÕES SOBRE A INFLUÊNCIA DOS MEIOS DE COMUNICAÇÃO NO CONSUMO DE BEBIDAS ALCOÓLICAS E SUA RELAÇÃO COM AS POLÍTICAS DE SAÚDE PÚBLICA
}

\author{
CIARLO, Thaís Bozelli \\ Psicóloga* formada pelo Centro Universitário de Araraquara - Uniara. \\ MASTROIANNI, Fábio de Carvalho \\ Psicólogo e Professor do curso de graduação em Psicologia e Direito do Centro Universitário de \\ Araraquara - Uniara. Av. Doutor Adhemar Pereira de Barros, 58, casa 28-Vila Melhado. CEP 14807-040. \\ Araraquara/SP. E-mail: psicomastroianni@gmail.com.br
}

\begin{abstract}
RESUMO
Problemas relacionados ao consumo de álcool são frequentes, especialmente entre os jovens. A publicidade de bebidas alcoólicas influencia o seu consumo e é um dos fatores passíveis de modificação. Neste artigo, foi realizada uma revisão bibliográfica de trabalhos que investigaram, sob diversos pontos de vista, a relação entre mídia e consumo. Utilizou-se a base de dados Scielo e o site de busca Google Acadêmico para artigoscientíficos, sendo adotados os termos mídia, consumo de álcool e propagandas, assim como livros de referência na área. A leitura dos textos nos permite refletir sobre a influência da mídia no consumo de álcool, podendo-se verificar a alta exposição dos jovens, tornando-se pertinente uma discussão sobre o tema, principalmente no que se refere às políticas de proteção, assim como das regras de auto-regulamentação publicitária frente às políticas públicas de saúde.
\end{abstract}

Palavras-chave: Mídia; Álcool, Saúde pública.

\begin{abstract}
Problems related to alcohol consumption are frequent, especially among young people. Alcohol beverage advertisements influence its consumption and is one of the liable to modification factors. In this work, it was carried out a bibliographical review of works that investigated, from several points of view, the relationship between media and consumption. We have used Scielo database and Google Academic for scientific works, searching for the keywords media, alcohol consumption and advertising, as well as articles and books of reference in the area. The reading of the texts has allowed us to reflect on the influence of media in alcohol consumption, and we can also verify the high exposition of youth to alcohol advertisements, becoming relevant a discussion on the subject, particularly regarding protection policies, as well as self-regulamentation advertising rules in public health policies.
\end{abstract}

KEYwords: Media; Alcohol; Public health.

*Este artigo é parte integrante do Trabalho de Conclusão de Curso para obtenção do título de Psicólogo. 


\section{INTRODUÇÃo}

O uso de drogas psicotrópicas e o consumo de álcool pela humanidade ocorrem desde a Antiguidade. Ao longo do tempo, esse consumo esteve associado a diversos aspectos e funções, entre eles o favorecimento da integração social. Na Idade Média o seu uso foi associado a comportamentos anti-sociais, mas voltou a ser estimulado a partir do Iluminismo, favorecendo a produção e comercialização do produto (CARLINI et al., 2009; ESCOHOTADO, 2000; SILVEIRA; MOREIRA, 2006; SCHULTES; HOFMAN, 1979). Já na metade do século XX, o uso dessas substâncias adotou claramente um caráter político-econômico, tornando-se nas últimas décadas um problema de saúde pública (MACRAE, 2001 apud GORGULHO, 2006).

Entre as principais substâncias consumidas em nosso país, o álcool apresenta uma das maiores prevalências; seu uso é lícito e o consumo é estimulado através da mídia, recebendo uma representação bastante diferenciada, quando comparado às outras drogas. Um estudo domiciliar realizado pelo Centro Brasileiro de Informações sobre Drogas Psicotrópicas (Cebrid) verificou que o álcool foi uma das drogas com maior prevalência de uso na vida $(68,7 \%)$, sendo as estimativas de dependentes de álcool em cerca de $6 \%$ (GORGULHO, 2006; CARLINI et al., 2002).

Estudos realizados com populações de estudantes do ensino fundamental e médio da rede pública de ensino, com idade entre 13 e 15 anos, em 27 capitais brasileiras, revelaram que cerca de $11,7 \%$ dos entrevistados eram usuários frequentes de álcool definido como o uso de seis vezes ou mais no último mês - e 6,7\% faziam uso pesado do álcool - definido como uso de 12 ou mais vezes de álcool nos últimos 30 dias (ANDRADE; ANDRADE; SILVA; SILVEIRA; SILVEIRA; SILVEIRA, 2008 apud GALDURÓZ et al., 2004).

Já um levantamento realizado com estudantes do ensino superior de duas faculdades do Estado de São Paulo revelou que $11,8 \%$ dos estudantes do sexo masculino e 1,3\% do sexo feminino foram classificados como bebedores-problema. Com relação à dependência de álcool, o estudo apontou índices de
$4,2 \%$ no sexo masculino e $0,8 \%$ no sexo feminino (BORINI; OLIVEIRA; MARTINS; GUIMARÃES, 1994 apud CAETANO; GALDURÓZ, 2004). Um outro estudo realizado com meninos em situação de rua (MOURA; SILVA; NOTO, 2009; CAETANO; GALDURÓZ, 2004) aponta que o uso de álcool na vida para essa população nas cidades de São Paulo $(86 \%)$ e Porto Alegre $(74,5 \%)$ tiveram um discreto aumento nos últimos anos. Além disso, o perfil de consumo dessa população é extremamente mais elevado quando comparados com os estudantes (GALDURÓZ, 2001).

Embora o uso de álcool possa ter ampla aceitação por grande parte da sociedade, o seu consumo excessivo está associado a inúmeras consequências, entre elas: acidentes de trânsito, aumento da violência, desenvolvimento da síndrome de dependência, além de ser responsável por $95 \%$ das internações hospitalares provocadas pelo uso de substâncias psicotrópicas (GALDURÓZ, 2001; CARLINI et al., 2009). Nesse contexto, o consumo de álcool torna-se um grande problema para a saúde pública, acarretando altos custos para a sociedade. Dados da Organização Mundial de Saúde (OMS) mostram que os gastos referentes ao consumo de bebidas alcoólicas representam $4 \%$ do custo total da saúde pública mundial; contudo, na América do Sul, esse índice varia entre $8 \%$ e $15 \%$ (CARLINI et al., 2009; FARIA; PINSKY; SILVA; VENDRAME, 2009).

Para identificar os padrões de consumo de cada país, a fim de fazer um cálculo do peso global dos danos causados relacionados ao álcool, a OMS caracterizou um padrão em cada nação do globo terrestre, classificando em quatro níveis de risco, com variação crescente de 1 a 4 . Um dado preocupante é que, no Brasil, se verificou a vigência de padrões de consumo com elevado grau de risco (nível 4). Os estudos demonstram também que o consumo de álcool é considerado 1 dos 10 comportamentos de maior risco à saúde, causando a morte de 1,8 milhões de pessoas no mundo $-5 \%$ delas, jovens entre 15 e 29 anos. As pesquisas ainda revelam que, para a população masculina, $5,6 \%$ de todas as mortes que 
ocorrem no planeta são atribuíveis ao consumo de álcool (WHO, 2002 apud GORGULHO, 2006; LARANJEIRA; MELONI, 2004).

Quando nos detemos à população adolescente, a questão relacionada ao uso de álcool vem preocupando cada vez mais os pesquisadores e profissionais das áreas de saúde e educação, uma vez que esse uso geralmente se inicia na adolescência e o consumo mundial de drogas, ao longo dos anos, vem aumentando consideravelmente (CARLINI, 1990; CARLINI; COTRIN, 1994 apud PERGHER; RIBEIRO; TOROSSIAN, 1998). Em oposição a esses aspectos, o consumo de bebidas alcoólicas em campanhas publicitárias é cada vez mais estimulado e tendenciosamente voltado para as populações mais jovens: com bebidas mais atraentes, sabores, embalagens e novas possibilidades de uso (GORGULHO, 2006). Em contraponto às propagandas, as campanhas de prevenção raramente abordam a questão dos problemas que o produto acarreta à saúde pública, como, por exemplo, ser responsável por $95 \%$ das internações hospitalares provocadas pelo uso de substâncias psicotrópicas (GALDURÓZ, 2001; CARLINI et al., 2009).

Estudos apontam que jovens com diferentes características podem interpretar de várias formas os anúncios de álcool. Algumas características são percebidas como atraentes para adolescentes entre 10 e 17 anos, como: o enredo da história, humor, personagens, evidenciando uma relação entre a apreciação pela propaganda e o aumento da intenção de comprar o produto. Considerando-se que a mídia constitui uma referência importante na sociedade atual, apresentando modelos e padrões de comportamento, podemos ponderar que ela também possui um importante papel no desenvolvimento dos jovens (PERGUER; RIBEIRO; TOROSSIAN, 1998; JUNDI; PINSKY, 2008).

Apesar das diversas consequências relacionadas ao consumo de bebidas alcoólicas, isso não impulsiona a criação de programas de controle ao álcool, como acontece com as drogas ilícitas, evidenciando, portanto, maior tolerância ao seu uso (CARLINI et al., 2009; ANDI, 2005). Outras drogas lícitas, como o tabaco e alguns medicamentos psicotrópicos (especialmente ansiolíticos e anfetaminas), embora não tão alardeados, continuam sendo as drogas mais consumidas e as que trazem os maiores prejuízos à população brasileira. Contudo, ainda são muito pouco consistentes as intervenções preventivas voltadas para essas drogas, deixando aberto o espaço para campanhas publicitárias cada vez mais sofisticadas para a promoção do consumo, que mascaram os inúmeros problemas sociais que envolvem o abuso do álcool (GALDURÓZ; NOTO, 1999).

Apesar de a indústria do álcool sustentar o argumento de que a propaganda é um instrumento de escolha de marcas para o indivíduo, alguns estudos acerca de políticas públicas sobre o álcool resumemo avanço feito nessa área, concluindo que, além da diferenciação entre as marcas, a campanha publicitária de álcool "reforça atitudes pró-álcool, pode aumentar o consumo de álcool entre indivíduos que já consomem, desencorajar bebedores a reduzir ou parar o consumo e influenciar a formação de políticas públicas" (p. 169) (EDWARDS; ANDERSON; BABOR; CASSWELL; FERRENCE; GIESBRECHT et al. apud PAVARINO FILHO; PINSKY, 2007).

Diante desse contexto, é válido mencionar a Lei Federal n. ${ }^{\circ}$ 9.294, de 1996, que traz regras mais específicas às propagandas de bebidas alcoólicas e regulamenta também medicamentos, produtos agrícolas e tabaco. Para efeito dessa lei, no entanto, as bebidas alcoólicas são apenas aquelas com um grau alcoólico superior a $13^{\circ} \mathrm{GL}$, ou seja, não incluindo a cerveja (sendo este o produto alcoólico com maior gasto em propaganda) e a maioria dos vinhos. Sua principal contribuição é restringir os horários de veiculação das propagandas entre 21 e 6 horas. No entanto, as vinhetas são permitidas em qualquer horário (CARLINI-COTRIM et al., 1995 apud GALDURÓZ; NOTO, 1998; PAVARINO FILHO; PINSKY, 2007).

Atentando-se para o impacto do consumo nocivo dessa substância à saúde, economia e segurança pública do país, recentemente a Agência Nacional 
de Vigilância Sanitária (Anvisa) lançou um edital para consulta pública com o objetivo de limitar as propagandas de bebidas alcoólicas, proposta esta que foi muito atacada pela indústria do álcool e da propaganda com argumentos em relação à liberdade de expressão (CARLINI-COTRIM et al., 1995 apud GALDURÓZ; NOTO, 1998; PAVARINO FILHO; PINSKY, 2007).

Segundo estimativas da Organização das Nações Unidas (ONU), os valores movimentados por esse comércio giram em torno de "400 bilhões de dólares anuais (maior que a indústria do petróleo), empregando perto de 20 milhões de pessoas e servindo de 70 a 100 milhões de consumidores" (COYLE, 2003 apud GORGULHO, 2006), tornando-se um alvo para o mercado da mídia, ainda que não se utilize esta como ferramenta para esclarecer, informar e facilitar a compreensão dos consumidores e, consequentemente, à consciência social que é influenciada pelos meios de comunicação (GORGULHO, 2006).

Todavia, quantias altíssimas são investidas na publicidade de produtos como bebidas alcoólicas, impulsionada pelo desenvolvimento tecnológico e avanços da globalização econômica e cultural. As pesquisas utilizadas acerca do comportamento humano e monitoramento das preferências dos consumidores são cada vez mais avançadas, demonstrando que não haveria tal investimento se não assegurassem o seu poder de influência. Além disso, a propaganda de bebidas alcoólicas propicia a criação de memórias afetivas positivas, ou "âncoras", portanto, o álcool passa a fazer parte da autoimagem, a constituir um estilo, uma maneira de ser, reforçando a escolha do produto (BABOR; CAETANO; CASSWELL; EDWARDS; GIESBRECHT, 2003 apud PAVARINO FILHO; PINSKY, 2007; JUNDI; PINSKY, 2008; GRAVAS, 2007 apud GORGULHO, 2006).

Estudos realizados com alunos do ensino médio demonstram que eles lembravam pouco ou nada de campanhas preventivas sobre álcool e drogas. Por unanimidade, os meios de comunicação em destaque eram a televisão, seguido de revistas com propagandas de prevenção ao uso de drogas. Pôdese constatar que a conscientização sobre as questões prejudiciais do uso de álcool e outras drogas não está sendo efetiva entre os adolescentes. Além disso, o álcool foi a substância psicotrópica mais abordada em campanhas publicitárias, segundo os sujeitos da pesquisa (COSTA; LUIS; OLIVEIRA; PILLON, 2002).

Diante desse contexto, o Conselho Nacional de Auto Regulamentação Publicitária (Conar) divulgou uma resolução, em outubro de 2003, definindo vários parâmetros restritivos à propaganda de bebidas alcoólicas, propondo: a exclusão de imagens voltadas para menores, suspendendo a participação de pessoas com menos de 25 anos nos comercias; desenhos animados; dentre outras normas estabelecidas. Apenas uma advertência é explicitamente voltada à informação de que o consumo não é destinado para crianças e adolescentes: "Este produto é destinado a adultos." No entanto, é discrepante o impacto da propaganda de bebida alcoólica, com belas imagens, seguida da tarja governamental, sóbria e obrigatória, informando sobre os danos causados pelo abuso da substância (PECHANSKY; SZOBOT; SCIVOLETTO, 2004).

Num país como o Brasil, onde a maioria da população tem a mídia como principal fonte de informações, o que é divulgado pelos meios de comunicação de massa passa a ser padrão de verdade. Além disso, não apenas a publicidade, mas os meios de comunicação, de modo geral, são importantes instrumentos na divulgação de informação. Esses veículos contribuem para a formação da opinião das pessoas, assim como também constituem um objeto de consumo, uma experiência individual cotidiana, um sistema de mediação cultural e de agregação social, além de tantas outras funções (WOLF, 2003).

De acordo com o autor, os efeitos dos meios de comunicação sobre as pessoas não devem ser compreendidos em modelos de curto prazo, mas sim de modo cumulativo e cognitivo. A mídia influencia o modo como o destinatário organiza a 
própria imagem do ambiente, desempenhando uma função de construção da realidade. Nesse contexto, surge a hipótese da agenda-setting, que pode ser entendida como a tendência que as pessoas têm de incluir ou excluir de seus próprios conhecimentos o que a mídia inclui ou exclui do seu próprio conteúdo. Desse modo, a mídia seleciona os conteúdos que devem ou não ser considerados importantes na agenda de acontecimentos (WOLF, 2003; TRAQUINA, 2005).

Se por um lado a imprensa não consegue dizer ao seu público o que pensar, por outro, ela é efetiva em dizer aos seus destinatários sobre quais temas pensar alguma coisa (WOLF, 2003; ATKIN; DEJONG, 2000). Sendo assim, quanto menor for a experiência direta e pessoal dos indivíduos sobre um determinado tema, mais ela dependerá dos meios de comunicação para obter as informações e os quadros interpretativos relativos àquela área (WOLF, 2003).

Os meios de comunicação, por meio do jornalismo, são capazes de introduzir conceitos gerais, informações básicas e podem também ajudar a estabelecer um clima de aceitação de questões críticas, constituindo-se numa fonte primária para prover a população com amplas mensagens. Na medida em que os jornais exercem um papel crítico no controle do acesso público à informação, a cobertura de notícias, junto com outras fontes primárias de socialização, podem influenciar o julgamento dos leitores, formando crenças e atitudes em relação a um tema (BAILLIE, 1996; MYHRE et al., 2002).

Estudos que buscaram analisar o papel da imprensa brasileira em relação ao tema das drogas (ANDI, 2005; MASTROIANNI, 2006; NOTO; PINSKY; MASTROIANNI, 2006) demonstram que, apesar de o jornalismo ter como função levar a discussão para a população, ampliando o conhecimento de seus leitores e estimulando reflexões, nem sempre isso ocorre em relação às drogas, uma vez que, tanto a análise das publicações, como do discurso dos profissionais da área, apontam para a superficialidade como o tema é tratado pelo jornalismo. Nesse sentido, entende-se que o modelo atual não tem oferecido à população e ao leitor maiores oportunidades de reflexão e discussão sobre o tema.

\section{ОвJеTIVo}

O presente trabalho tem como objetivo analisar pesquisas científicas e textos existentes sobre os temas: mídia, propagandas de bebidas alcoólicas e consumo de álcool, visando compreender a relação entre esses fatores e o modo como os veículos de comunicação podem influenciar o comportamento humano. Aleitura do presente artigo também busca oferecer uma reflexão sobre o tema, tendo em vista a sua atualidade e as diversas consequências oriundas do consumo excessivo de álcool na sociedade contemporânea.

\section{Metodologia}

Realizou-se revisão bibliográfica utilizando-se a base de dados Scielo, bem como o site de busca Google Acadêmico para artigos científicos - neste último, selecionados para estudo apenas os trabalhos científicos. Os critérios de seleção dos artigos basearam-se nos seguintes termos: mídia, consumo de álcool e propagandas, sendo eles revisados na íntegra, assim como também se privilegiou a utilização de livros de importante referência na área, conforme observado na literatura.

A busca de artigos de pesquisa foi feita para o período compreendido entre 1995 e 2009, em língua portuguesa. Todavia, foram incluídos alguns trabalhos de autores internacionais, traduzidos para o português, e artigos científicos publicados na língua inglesa, pois estes foram considerados pertinentes para contextualizar as informações obtidas no trabalho. Privilegiou-se, na seleção dos artigos estudados, aqueles contendo estudos empíricos, epidemiológicos, conceituais e de revisão que relacionassem o consumo de álcool e/ou a influência da mídia. Durante a coleta de material, foram encontrados sete artigos de acordo com os critérios de seleção adotados, todos eles analisados e indicados nas referências. 


\section{Resultados E DiscuSSÃo}

Os estudos, pesquisas e teorias revisadas neste trabalho demonstram que existe uma relação entre consumo de bebidas alcoólicas e o modo como as mensagens sobre esse tema são veiculadas nos meios de comunicação. Embora este estudo se concentre apenas na revisão de artigos e pesquisas, a leitura crítica dos textos favorece a reflexão e propõe uma discussão acerca da problemática envolvendo o uso de bebidas alcoólicas, além das consequências oriundas desse consumo nos programas de saúde pública.

Observa-se que o consumo de drogas psicotrópicas vem aumentando em todo o mundo; o álcool, por sua vez, é a droga mais consumida no país, tendo aceitação por grande parte da sociedade, principalmente no que se refere às populações mais jovens. Seu consumo excessivo ou inadequado está relacionado a inúmeras consequências negativas, tornando-se um grande problema de saúde pública. Além disso, verifica-se que a realização de esforços no sentido de minimizar ou reverter essa situação demandam altos gastos e investimentos do setor público.

Em contraste com esse quadro, verifica-se que as propagandas desse produto, apesar das restrições e regulamentações acerca de sua divulgação, são cada vez mais criativas e voltadas à população juvenil. Além disso, estudos e teorias sobre comunicação de massa apontam que a mídia tem o potencial de estabelecer padrões, influenciar comportamentos e lançar novas tendências, exercendo, portanto, um importante papel no desenvolvimento dos jovens. Nesse contexto, percebe-se que, enquanto as propagandas incentivam o consumo, os demais meios de comunicação, como o jornalismo, por exemplo, não conseguem, de forma satisfatória, discutir e oferecer à população um debate mais consciente sobre a problemática.

Analisando a relação dos meios de comunicação com as políticas de saúde, observa-se que as campanhas de prevenção e os programas de controle ao uso de substâncias psicotrópicas parecem ser mais direcionados às drogas ilícitas, ainda que o maior consumo esteja relacionado às drogas lícitas, como o álcool. Essas questões colocam o consumo de bebidas alcoólicas em uma posição bastante complexa, pois, se de um lado o álcool é a droga mais utilizada, acarretando diversos gastos e consequências negativas, principalmente entre os jovens, por outro, é a substância psicotrópica mais disseminada e estimulada pelos meios de comunicação.

Essa dinâmica nos leva a pensar que a mídia, principalmente no que se refere às propagandas de bebidas alcoólicas, segue na contramão dos objetivos e necessidades de políticas de saúde pública. Além disso, é válido destacar que esse incentivo indiscriminado ao consumo se contrapõe também aos preceitos do Estatuto da Criança e do Adolescente (ECA - Lei 8.069/90), que dispõe sobre a proteção integral à criança e ao adolescente, sendo dever da família, da comunidade, da sociedade em geral e do poder público assegurar, com absoluta prioridade, os seus direitos, evitando que sejam objetos de qualquer forma de exploração ou negligência. $\mathrm{O}$ artigo 81 dessa lei, em seu inciso 2, deixa bastante clara a restrição dessa população ao acesso e à comercialização de bebidas alcoólicas.

Faz-se necessário salientar que, em hipótese alguma, a leitura dos textos e pesquisas sugere que os meios de comunicação são os principais responsáveis pelos problemas oriundos do consumo excessivo de álcool. Em vez disso, a análise dos estudos revisados neste artigo nos permite observar não só a importância e o potencial desses instrumentos, como também a possibilidade de se utilizar esses recursos associados às campanhas de prevenção, entre outras atividades voltadas às políticas de saúde pública. Exemplos disso são algumas campanhas recentemente lançadas pelo Ministério da Saúde, em que as mesmas técnicas utilizadas pelos publicitários, visando estimular o consumo, são usadas para levar à população o conhecimento sobre os riscos e a necessidade de se refletir sobre o problema, apresentando realidades 
que as propagandas de incentivo não mostram.

De qualquer forma, é muito difícil imaginar um programa de prevenção - ou de controle - ao consumo de substâncias psicotrópicas, com o objetivo de informar e alertar a população, que não utilize os recursos e os benefícios oferecidos pelos meios de comunicação. No que se refere à questão do álcool, o que se observa é que há simultaneamente a ação de duas forças antagônicas, ou seja, ao mesmo tempo em que a mídia pode ser utilizada para prevenir, também é usada para estimular o consumo. Além de favorecer sentimentos e percepções contraditórias em relação ao tema, podendo gerar confusões, a utilização das propagandas em duas vias distintas também não permite a modificação da problemática atual.

Identificando essas questões e visando alterar esse quadro, eliminando a ação de forças em sentidos opostos, alguns movimentos buscam restringir o incentivo do consumo de bebidas alcoólicas na imprensa. Um exemplo é o movimento organizado por membros da própria sociedade, denominado "propaganda sem bebida" (http:// www.propagandasembebida.org.br/), que tem como uma de suas metas aprovar lei que limita a publicidade de bebidas alcoólicas nos meios de comunicação e em eventos esportivos, culturais e sociais, semelhante à legislação atual, que restringe as propagandas de tabaco que entraram em vigor no início da década.

A reflexão proporcionada pelas leituras não direciona a discussão para questões referentes à aprovação ou não do consumo de bebidas alcoólicas, tampouco ao impedimento da comercialização do produto. Possibilita, no entanto, pensar sobre as vantagens ligadas em se eliminar ou restringir variáveis que não colaboram com os esforços e metas das políticas de saúde pública, como as propagandas que incentivam o consumo e agem no sentido inverso, gerando contradição e confusões sobre um tema tão delicado.

Por fim, vale destacar que apenas isso não reverterá a situação atual, mas que, em médio e longo prazos, algumas mudanças poderão ser observadas. É importante considerar que, havendo informação adequada que permita à população refletir e pensar sobre o tema, esta também poderá influenciar e participar da construção de uma política pública mais efetiva, posicionando-se frente às variáveis que possam interferir ou prejudicar o seu bem-estar social.

\section{CONSIDERaÇões Finais}

Mais do que confirmar, rever ou questionar a influência da mídia sobre o comportamento das pessoas ou verificar seu efeito sobre a sociedade, este artigo buscou favorecer uma discussão sobre a importância de se atentar para os benefícios oferecidos pelos meios de comunicação, refletindo de que modo eles podem ser úteis em programas e políticas de saúde pública, mais especificamente sobre o álcool. Outras pesquisas que busquem explorar a relação entre sociedade e políticas públicas, assim como sua ligação com os meios de comunicação, poderão ampliar ainda mais a discussão sobre um tema que é tão delicado, uma vez que interesses econômicos acabam se chocando com problemas de saúde pública.

\section{REFERÊNCIAS}

ANDI (Agência Nacional dos Direitos da Infância). Mídia e Drogas, Cortez, São Paulo, 2005. 80p.

ANDRADE, A. G.; ANDRADE, H. S. G.; SILVA, J. G.; SILVEIRA, C. M., SILVEIRA, C. C.; SILVEIRA, L. M.; Epidemiologia do beber pesado e beber pesado episódico no Brasil: uma revisão sistemática da literatura. São Paulo. 2008. Disponível em: http://www.scielo.br/scielo.php? script=sci_arttext\&pid=S010160832008000700008 \&lang=pt. Acesso em: 18 jun 2009.

ATKIN, C.K. \& DEJONG, W. News coverage of alcohol and other drugs in U.S. college newspapers. Journal of Drug Education, v. 30, n. 4, p. 453-465, 2000. 
BAILLIE, R.K. Determining the effects of media portrayals of alcohol: going beyond short term influence. Alcohol and Alcoholism, v. 31, n.3, p. 235-242, 1996.

CAETANO, R.; GALDURÓZ, J. C. F., Epidemiologia do uso de álcool no Brasil - Revista Brasileira de Psiquiatria; 2004; 26 (Supl 1): 3-6. Disponível em: http://www.scielo.br/scielo.php?script= sci_arttext\&pid=S151644462004000500002\&lang=pt. Acesso em: 9 jun. 2009.

CARLINI, E.A.; GALDURÓZ, J.C.F.; NOTO, A.R.; NAPPO, S.A. I Levantamento Domiciliar Nacional sobre o uso de Drogas Psicotrópicas, São Paulo: CEBRID, UNIFESP, 2002. 380p.

COSTA, L. A.; LUIS, M. A. V.; OLIVEIRA, P. P.; PILLON, S. C. O que os estudantes captam nas propagandas sobre álcool e drogas: um estudo piloto. Simpósio Brasileiro de Enfermagem, 2002. Disponível em: http://www.proceedings.scielo.br/ scielo.php?pid=msc0000000052002000100041\& script=sci_arttext\&tlng=pt. Acesso em: 8 jun. 2009.

ESCOHOTADO, A. Historia general de lãs drogas. 3.ed. Madrid: Espasa, 2000.

FARIA, R.; PINSKY, I.; SILVA, R.; VENDRAME, A. Apreciação de propagandas de cerveja por adolescentes: relações com a exposição prévia às mesmas e o consumo de álcool, Rio de Janeiro; 2009. Disponívelem: http://www.scielo.br/scielo.php? pid=S0102-311X2009000200014\&script $=$ sci_abstract\&tlng=pt. Acesso em: 7 jul. 2009.

GALDURÓZ, J. C. F.; NOTO, A. R. O uso de drogas psicotrópicas e a prevenção no Brasil, Rio de Janeiro, 1999. Disponível em: http://www.scielosp. org/scielo.php?pid=S1413-81231999000100012 \&script=sci_arttext. Acesso em: 2 jun. 2009.

GALDURÓZ, J. C. F. O Uso e Abuso de Drogas
Psicotrópicas no Brasil. Revista IMESC, n. ${ }^{\circ} 3$, p. 37-42. 2001. Disponível em:

http://www.revistasusp.sibi.usp.br/scielo.php?pid= S010411692005000700017\&script=sci_arttext $\&$ tlng=pt. Acesso em: 2 agos. 2009.

GORGULHO, M. A Influência as Mídia na Realidade Brasileira do Fenômeno das Substâncias Psicoativas. In:

Panorama Atual de Drogas e Dependências. São Paulo: Atheneu. 2006. Cap. 19.

JUNDI, S. A. R. J. EL.; PINSKY, I. O impacto da publicidade de bebidas alcoólicas sobre o consumo entre jovens: revisão da literatura internacional, São Paulo, 2008. Disponível em: 〈http://www.scielo. br/pdf/rbp/v30n4/213pdf>. Acesso em: 2 out. 2010.

LARANJEIRA, R.; MELONI, J. N. Custo social e de saúde do consumo de álcool, São Paulo, 2004. Disponível em: <http://www.scielo.br/scielo.php? pid $=$ S1516-44462004000500003\&script $=$ sci_abstract\&tlng=pt>. Acesso em: 7 jul. 2009.

LEMMENS, P.H., VAETH, P.A.C., GEENFIELD, T.K. Coverage of Beverage Alcohol Issues in the Print Media in the United States, 1985-1991. American Journal of Public Health, v. 89, n. 10, p. 15551560, 1999.

MASTROIANNI, F. C. As drogas psicotrópicas e a imprensa brasileira: análise do material publicado e do discurso dos profissionais da área de jornalismo, 2006. Dissertação (Mestrado) Universidade Federal de São Paulo - Departamento de Psicobiologia, 2006.

MOURA, Y. G. ; SILVA, E. A. ; NOTO, A. R. . Redes Sociais no contexto de uso de drogas entre crianças e adolescentes em situação de rua. Psicologia em pesquisa. v. 3, p. 31-46, 2009.

MYHRE, S., SAPHIR, M.N., FLORA, J.A., 
HOWARD, K.A., GONZALES, E.M. Alcohol Coverage in California Newspaper: frequency, prominence and framing. Journal of Public Health Policy, v. 23, n. 2, p. 172-190, 2002.

NOBLAT, R. A arte de fazer um jornal diário. São Paulo, Contexto, 2003. 173 p.

NOTO, A.R., PINSKY,I., MASTROIANNI, F.C. Drugs in the brazilian print media: An exploratory survey of newspaper and magazine stories in the year 2000, Substance Use and Misuse, v. 41, p. 1263-1276, 2006.

PAVARINO FILHO, R. V. P.; PINSKY, I. A apologia do consumo de bebidas alcoólicas e da velocidade no trânsito no Brasil: considerações sobre a propaganda de dois problemas de saúde pública, Rio Grande do Sul, 2007. Disponível em: <http:// www.scielo.br/scielo.php?pid=S010181082007000 $100019 \&$ script=sci_arttext\&tlng=end_0100-6916>. Acesso em: 7 jul. 2009.

PAVARINO FILHO, R. V. P.; PINSKY, I. A apologia do consumo de bebidas alcoólicas e da velocidade no trânsito no Brasil: considerações sobre a propaganda de dois problemas de saúde pública, Rio Grande do Sul, 2007. Disponível em: <http:// www.scielo.br/scielo.php?pid=S010181082007000 100019\&script=sci_arttext\&tlng=end_0100-6916> . Acesso em: 7 jul. 2009.

PECHANSKY, F.; SCIVOLETTO, S.; SZOBOT, C. M.; - Uso de álcool entre adolescentes: conceitos, características epistemológicas e fatores etiopatogênicos, Revista Brasileira de Psiquiatria, 2004. Disponível em: <http://www.scielo.br/scielo.

php ? script =sci_arttext \& pid=S $1516-$ 44462004000500005>. Acesso em: 2 set. 2009.

PERGHER, N. K.; RIBEIRO, T. W.; TOROSSIAN, S. D. Drogas e adolescência: uma análise da ideologia presente na mídia escrita destinada ao grande público, Porto Alegre, 1998. Disponível em: http://www. scielo.br/scielo.php?pid=S0102$79721998000300003 \&$ script=sci_arttext\&tlng=in. Acesso em: 10 jun. 2009.

SCHULTES, E.R. \& HOFMANN, A. Plantas de Los Dioses: origenes del uso de los alucinógenos, México, 1979. 208 p.

TRAQUINA, N. Teorias do Jornalismo. 2.ed. Florianópolis: Insular, 2005. 224 p.

WOLF, M. Teoria das Comunicações de Massa. São Paulo, Martins Fontes, 2003. 295 p. 